\title{
Adaptive monogenic filtering and normalization of ESPI fringe patterns
}

\author{
J. A. Guerrero, J. L. Marroquin, and M. Rivera \\ Centro de Investigación en Matemáticas, Apdo. Postal 402, Guanajuato, Gto. 36000, Mexico \\ J. A. Quiroga \\ Departamento de Optica, Universidad Complutense de Madrid, 2841 Madrid, Spain
}

Received June 1, 2005; revised manuscript received July 22, 2005; accepted July 24, 2005

\begin{abstract}
A technique is presented for filtering and normalizing noisy fringe patterns, which may include closed fringes, so that single-frame demodulation schemes may be successfully applied. It is based on the construction of an adaptive filter as a linear combination of the responses of a set of isotropic bandpass filters. The space-varying coefficients are proportional to the envelope of the response of each filter, which in turn is computed by using the corresponding monogenic image [Felsberg and Sommer, IEEE Trans. Signal Process. 49, 3136 (2001)]. Some examples of demodulation of real Electronic Speckle Pattern Interferometry (ESPI) images patterns are presented. (C) 2005 Optical Society of America

OCIS codes: $100.2000,120.2650,120.6160$.
\end{abstract}

The demodulation of single-frame fringe patterns containing closed fringes is an interesting problem that has applications, for example, when the fringes are used to study fast transient phenomena. The difficulty of this problem stems from the fact that, although the absolute value of the local phase may be estimated from local (linear) operators-e.g., from the normalized intensity gradient-the determination of the phase sign requires tracking the local direction across the image and is therefore a global property ${ }^{1-4}$ For this reason demodulation algorithms are very sensitive to noise and local contrast variations, since even a small bad region may send the algorithm off track and have disastrous global consequences. This is true even when robust schemes, like the ones in Refs. 1 and 4, are used for the demodulation of real noisy patterns, such as the ones obtained from ESPI. Our purpose in this paper is to present an algorithm for producing, from real ESPI patterns with strong contrast variations, clean, normalized patterns that may be demodulated with existing procedures.

If one has a locally monochromatic pattern, i.e., a pattern that locally looks like $A \cos \left(\omega_{0}^{T} x+\phi\right)$, where $A$, $\omega_{0}$, and $\phi$ are constants, corrupted with a white (wideband) noise process, a narrowband bandpass filter tuned at $\omega_{0}$ will effectively eliminate noise; moreover, if one uses a quadrature filter pair (such as a complex-valued Gabor filter), one may obtain the local contrast $A$ as the magnitude of the complex filter output and, hence, normalize the pattern by dividing it by $A$. However, if the local frequency has a substantial variation across the image, a single filter will produce unreliable results in most places.

In theory, one may construct an adaptive filtering scheme by selecting a set of Gabor filters whose responses cover one half of the frequency plane and constructing the output as a weighted combination of the real part of the responses of each filter, where the weights depend on the amplitude of the corresponding response; the problem is that, since the passband of the filters must be narrow enough to have good noise reduction, this scheme would require too many filters to be of practical use.

As an alternative, one may use isotropic bandpass filters that have annular frequency responses (see Fig. 1a), so that the frequency plane may be covered by using only a small set (say, five) of them. In this case, to obtain the local amplitude, one must use two companion filters whose frequency responses have odd symmetry (Figs. 1b and 1c). In particular, one may express the two-dimensional frequency vector $\omega=\left(\omega_{1}, \omega_{2}\right)^{T}$ in polar coordinates $(\rho, \theta)$, so that $\omega$ $=(\rho \cos \theta, \rho \sin \theta)^{T}$; in this case, the three filters of Fig. 1 may be expressed in polar-separable form as

$$
\begin{gathered}
H_{0 k}(\rho, \theta)=G\left(\rho-\rho_{k}\right), \\
H_{1 k}(\rho, \theta)=-i G\left(\rho-\rho_{k}\right) \sin (\theta), \\
H_{2 k}(\rho, \theta)=-i G\left(\rho-\rho_{k}\right) \cos (\theta),
\end{gathered}
$$

where $G(\rho)$ is a bell-shaped function and $\rho_{k}$ is the tuning frequency. The vector-valued function $H_{k}$ $=\left(H_{0 k}, H_{1 k}, H_{2 k}\right)^{T}$ is the frequency response of the $k \mathrm{th}$ monogenic filter, and the vector-valued image $F_{k}$ $=\left(F_{0 k}, F_{1 k}, F_{2 k}\right)^{T}$ that is obtained as the output of this filter is called the monogenic image. ${ }^{5}$ One may easily verify that if a fringe pattern is locally monochromatic at pixel $x$, with local frequency $\omega_{0}$ $=\left(r_{0} \cos t_{0}, r_{0} \sin t_{0}\right)^{T}$, the $k$ th isotropic filter output

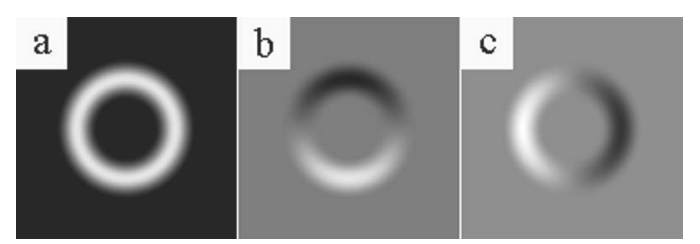

Fig. 1. a, Frequency response of an isotropic bandpass filter; $b, c$, imaginary part of the frequency response of the companion odd components of the monogenic filter. 


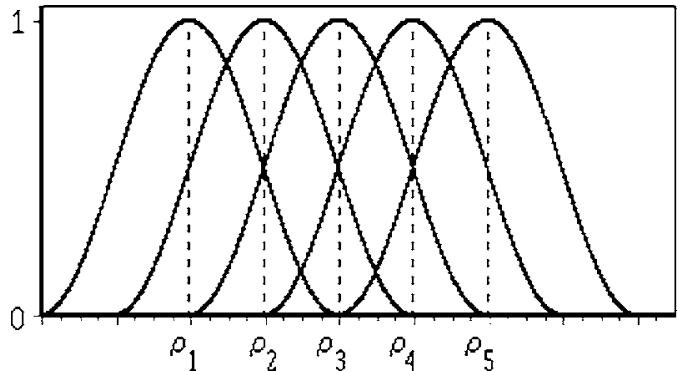

Fig. 2. Magnitude of the response of the set of monogenic filters as a function of frequency magnitude.

will be $F_{0 k}(x)=G\left(r_{0}-\rho_{k}\right) A \cos \left(\omega_{0}^{T} x+\phi\right)$, and the $k$ th monogenic image at this pixel will be a vector with magnitude $M_{k}(x)=G\left(r_{0}-\rho_{k}\right) A$, irrespective of the orientation angle $t_{0}$. If one takes $K$ monogenic filters whose overlapping frequency responses cover the region of interest in the frequency plane (see Fig. 2), one may construct an adaptive filter whose output is a linear combination of the responses of the isotropic filters $\left\{H_{0 k}, k=1, \ldots, K\right\}$, with coefficients (weights) that depend on the corresponding magnitudes. The same weights may be used to find an equivalent magnitude that normalizes this output, so that one finally obtains the normalized pattern $F$ as

$$
F(x)=\frac{\sum_{k=1}^{K} w_{k}(x) F_{0 k}(x)}{\sum_{k=1}^{K} w_{k}(x) M_{k}(x)} .
$$

The weights $w_{k}$ are computed as

$$
w_{k}(x)=\left(\frac{M_{k}(x)}{M_{\max }(x)}\right)^{p},
$$

where $M_{\max }(x)=\max _{k} M_{k}(x)$ and $p$ is a positive constant chosen so that $w_{k}(x)$ is close to 1 if $M_{k}(x)$ $\approx M_{\text {max }}(x)$ and is close to 0 otherwise. We have found that the results are for practical purposes insensitive to the precise value of $p$, as long as it is large enough (e.g., $p>5$ ). We have used $p=10$ in all the experiments reported here.

It is important to note that in ESPI patterns, since speckle noise is multiplicative, clear fringes are more affected by it than are dark ones. Since the filters we are proposing are linear-which implies that they treat clear and dark fringes in the same way-it is necessary to preprocess the image to reduce noise over clear fringes. One simple way to do this is by means of a max filter, whose output at pixel $x$ is defined as

$$
I_{M}(x)=\max _{y \in W(x)} I(y),
$$

where $I$ is the input image and $W(x)$ is a $3 \times 3$ window centered at pixel $x$. This filter has the effect of approximating the (upper) envelope of the pattern and hence reducing noise over the clear fringes. It also has the unwanted effect of reducing the local contrast, but this is effectively counteracted by the adaptive filtering and normalization implemented by Eq. (2).
The detailed implementation of the monogenic filters is as follows: first, the frequency dynamic range $[\alpha, \beta]$ of the input image (in radians/pixel) is found by setting $\alpha=\pi / \max w$ and $\beta=\pi / \min w$, where $\max w$, $\min w$ are the widths (in pixels) of the widest and the narrowest fringes of the pattern, respectively. We have developed a graphical interface with which these values may be easily specified by the user by pointing at the limits of the desired fringes. Of these two values, the most critical is max $w$ : if it is too large, some narrow fringes may be lost in the reconstruction, and if it is too small, noise elimination over wide fringes may be incomplete. Note, however, that if this filtering method is used in conjunction with a robust demodulation algorithm, such as in Ref. 4, this residual noise will be inconsequential for the final result, since it will be eliminated in the demodulation phase. In practice, one finds that underestimating $\max w$ to as low as $80 \%$ of its true value still produces good results, which means that it is safer to underestimate it slightly in the interactive procedure. Note that these are the only parameters that the user has to specify. Next, for the bell-shaped function $G$ we use

$$
\begin{aligned}
G(\rho) & =\frac{1}{2}\left[1+\sin \left(\frac{(h+2 \rho) \pi}{2 h}\right)\right] & & \text { for } \rho \in[-h, h], \\
& =0 & & \text { otherwise }
\end{aligned}
$$

where $h=(\beta-\alpha) /(K-1)$ (we take $K=5$ filters in all cases). The filters are then computed by using Eqs. (1), with $\rho_{k}=\alpha+h(k-1), k=1, \ldots, K$. (When $\alpha$ is very small, Eq. (4) has to be modified slightly to guarantee that the responses of all filters are 0 for $\rho=0$.) Filtering is performed in the frequency domain, and the output magnitudes $\left\{M_{k}\right\}$ are smoothed with a Gaussian kernel (with $\sigma=6$ ) before the weights are computed by use of Eq. (3).

In Fig. 3 we present the results of the monogenic adaptive filtering and normalization of a sequence of ESPI patterns that correspond to the thermal deformation of a computer monitor. The processing time for the complete filtering and normalization procedure, for a $1024 \times 1024$ pixel image, was $50 \mathrm{~s}$ on a $3 \mathrm{GHz}$ workstation. To illustrate how this filtering effectively helps in the demodulation process, we also include the output (wrapped phase) of a state-of-theart robust demodulation procedure, namely, the one in Ref. 4, applied to these filtered and normalized patterns. This method, which is, to our knowledge, the most robust demodulation procedure available for closed-fringe interferograms, starts by estimating the phase in a subregion of the image with relatively high-quality open fringes and iteratively extends this region by using a variational approach, along directions where the fringes are better defined (highestquality regions). In this way the phase in difficult regions, such as saddle points or places where the fringes are too noisy or have gaps, are left undefined until there is enough information around them to interpolate reasonable values. As one can see, this de- 


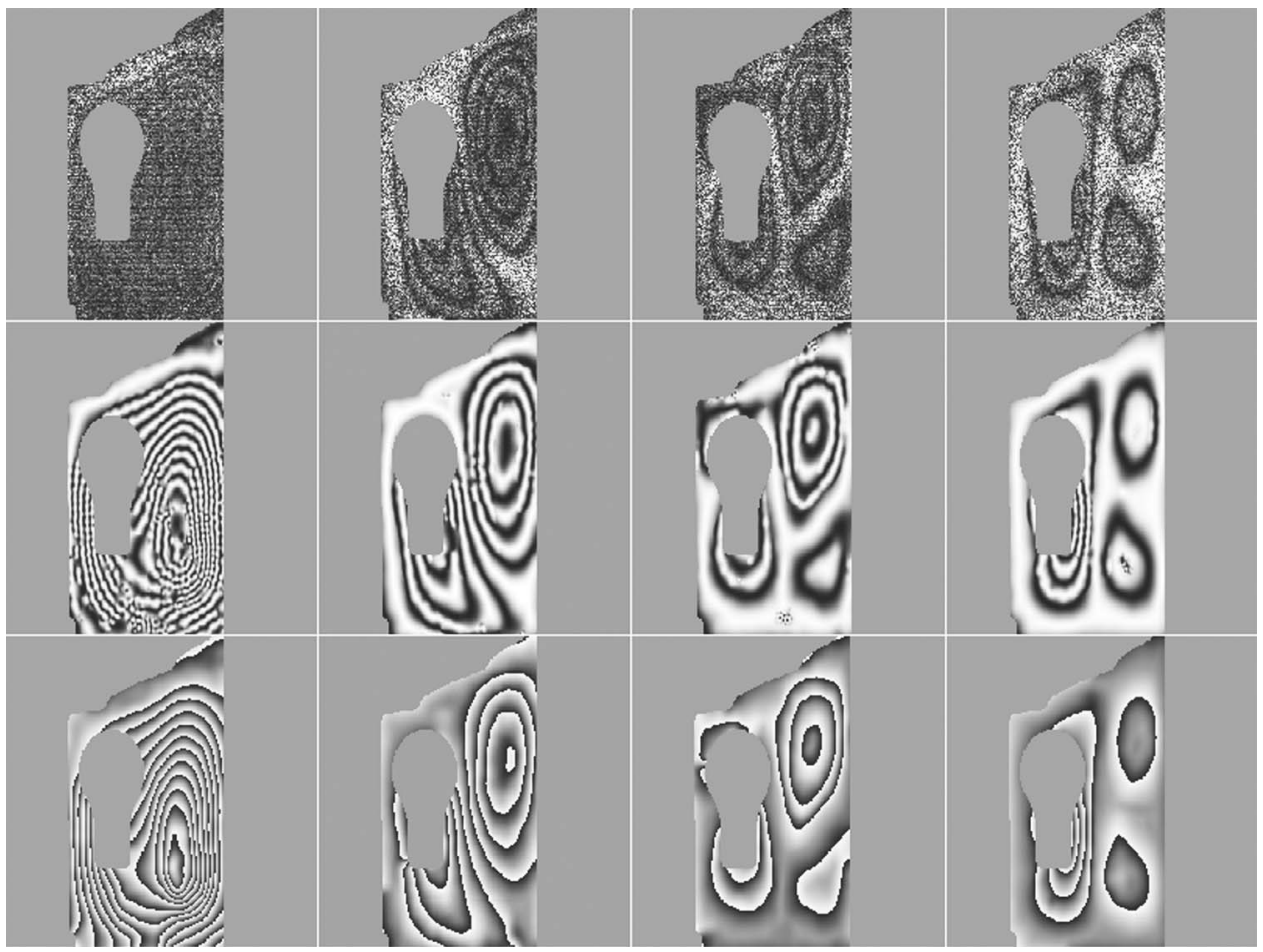

Fig. 3. First row, sequence of ESPI patterns. Second row, filtered and normalized images. Third row, output of a robust demodulation procedure (wrapped phase) applied to the second row.

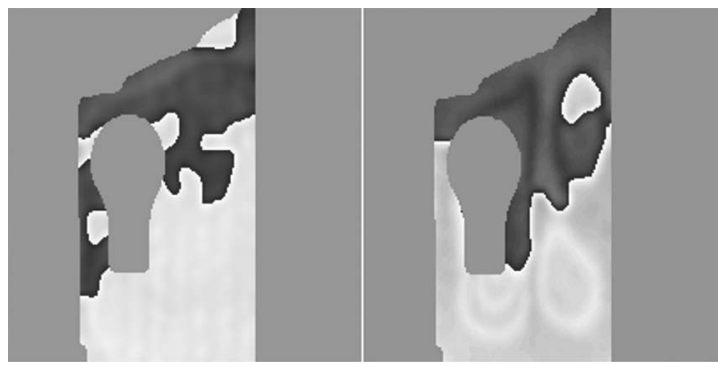

Fig. 4. Output (wrapped phase) of the same robust demodulation procedure applied to the first and last images of the first row of Fig. 3 .

modulation method is capable of recovering the correct phase, even when the fringes produced by the proposed preprocessing step are noisy or incomplete in small regions; if a simpler filtering and normalization preprocessing is applied, however, the method will fail. Thus, in Fig. 4 we show the output of the same demodulation procedure applied to the first and last input images (first row of Fig. 3) after smoothing them with a Gaussian kernel and rescaling their intensity to the interval $[-1,1]$. (The results for the other images of the sequence are similar.) As one can see, the standard filtering and normalization applied in this case cannot prevent the demodulation algorithm from getting an incorrect reconstruction (note that the recovered wrapped phase does not show $2 \pi$ jumps at each fringe, as expected), which means that the proposed filtering and normalization procedure effectively increases the range of applicability of demodulation schemes for closed-fringe ESPI patterns and may be very useful as a preprocessing step for other fringe pattern processing algorithms as well.

J. L. Marroquin (jlm@fractal.cimat.mx) and M. Rivera were supported in part by grants 46270 and 40722 from the Consejo Nacional de Ciencia y Tecnología, Mexico; J. A. Quiroga was supported by grant DPI2002-02104 from the Ministerio Español de Ciencia y Tecnología.

\section{References}

1. M. Servin, J. L. Marroquin, and J. A. Quiroga, J. Opt. Soc. Am. A 21, 411 (2004).

2. K. G. Larkin, D. J. Bone, and M. A. Oldfield, J. Opt. Soc. Am. A 18, 1862 (2000).

3. J. Kozlowsky and G. Serra, Appl. Opt. 38, 2256 (1999).

4. M. Rivera, J. Opt. Soc. Am. A 22, 1170 (2005).

5. M. Felsberg and G. Sommer, IEEE Trans. Signal Process. 49, 3136 (2001). 